\title{
Magnetorheological finishing of small gear teeth profiles using novel workpiece fixture
}

\section{DOI : 10.36909/jer.ICCEMME.15759} \author{
781039, India. \\ **National Institute of Technology Patna, Bihar, 800005, India. \\ *** Corresponding Author: manje176103021@iitg.ac.in
}

Manjesh kumar****, Abhinav Kumar*, Anupam Alok**, and Manas Das*

*Department of Mechanical Engineering, Indian Institute of Technology Guwahati, Assam,

\begin{abstract}
Miniature gears are essential components of transmitting power in tiny motors used in the aviation, automobile, and healthcare sectors etc. Because of the intricacy of its shape, nanofinishing of tiny gear is a tough job. The rotational magnetorheological abrasive flow finishing (R-MRAFF) technique is a new hybrid methodology for the generation of nanometer range surface finishing. These surfaces reduce friction between integrating parts, extending their life span. In the current study, a model for simulating the impacts of the RMRAFF technique was developed using finite element (FE) analysis software, namely COMSOL® Multiphysics. The impacts of various process factors on the fluid flow characteristics while finishing the gear component are investigated using magnetostatic fluid flow analysis of magnetorheological polishing fluid (MRPF) in 3D computational domain of new workpiece fixture. To evaluate the forces operating in R-MRAFF technique, a viscosity model for MRP fluid flow around a complicated component (small steel gear) in an outside magnetic field is recognized and simulated. The magnetic field has a major impact on processing effectiveness by controlling the MRPF viscosity. During the polishing of the gear component, the surface finish attained at various places on working surfaces is uniform, which is confirmed by surface characterization of teeth profiles of small gear.
\end{abstract}

Keywords: Finite element analysis; magnetorheological polishing fluid; rotational magnetorheological abrasive flow finishing process; nanofinishing; small gear; magnetic field. 


\section{INTRODUCTION}

As nanotechnology gains popularity, smaller components are becoming more in demand. In these tiny machines, which are used in sectors including aviation, automobile, military, and healthcare, small gears are essential for transmitting power and other equipment (Kumar et al., 2021). Nano-scale surface finish on the gear teeth allows power to be transferred smoothly, helps with noise-free operating, and contributes to extended service life. Tiny gears exhibit various kinds of manufacturing flaws including burr, dent, fractures, pit, and other types of defect (Yi et al., 2009). The burr degrade gear performance. It's hard to polish small gears in a traditional method. Conventional polishing methods, including as honing, lapping, grinding, and buffing, are not always effective at generating the detailed, precise results that are often required, such as in the case of complex 3D components, freeform shapes, and uneven structures (Alaskari et al., 2014). The different material properties like yield strength, wear, and corrosion resistance can be improved by super finishing. Hybrid processes like pulse electrochemical mechanical polishing (PECMP) are used to finish macro gears but not suitable for small gears (Yi et al., 2009). These methods have frequently proven insufficient to meet the requirements of contemporary healthcare, automobile, and military sectors. The area of nano-finishing addressed by these industries is not effective because of the inadequacy of these technologies.

An unconventional approach is R-MRAFF method, which is more precise than other polishing techniques (Kumar et al., 2020). Finishing is done by moving abrasives over the part surfaces in this technique (Nagdeve et al., 2018). For polishing stainless steel workpiece, a finishing process that involves a finishing medium with $\mathrm{Fe}$ powder and $\mathrm{SiC}$ abrasives as 26.5 Vol. \% Fe powder and 13.6 Vol. \% SiC was employed, and a best roughness of $17 \mathrm{~nm}$ was achieved (Soler and Le Nguyen, 2018). Material extraction is accomplished via the development of shear force among the abrasives and the working surfaces (Kumar et al., 2021). The key characteristic of the present method is that the fine surface polish is consistent. AFM can polish complex geometries but it is unable to be controlled in actual 
environment. In-contrast, R-MRAFF is easier to operate and it correspondingly offers more control over final product characteristics. A simpler version of the R-MRAFF model has been developed and is appropriate for use on flat surfaces when the magnetic field is oriented in a single direction.

From the previous study, it is observed that no magnetorheological-finishing of small gear teeth profiles has yet been reported, and also very few studies have been investigated on the simulation of non-Newtonian fluid on this type of complex profiles of small gear in which the cross-section area varies in the path of flow. In the present experimental investigation, a unique fixture is developed for the uniform finishing of small gears in the R-MRAFF process. The purpose of the present research to provide a more comprehensive theoretical understanding of the R-MRAFF technique, as well as a fundamental understanding of the nature of MRPF during surface polishing, via use of the FEA tool provided by COMSOL® Multiphysics software. SS316L metal is utilized to make tiny gear. The polished gear profile is inspected by using an optical profilometer and FESEM analysis. The finished small gear teeth profiles have no manufacturing defects while using a unique fixture in the R-MRAFF process.

\section{EXPERIMENTAL SETUP AND MAGNETOSTATIC FLUID FLOW FINITE ELEMENT ANALYSIS}

A three-dimensional computational domain (Figure 1) is utilized in the COMSOL® Multiphysics programme for the magnetostatic fluid flow study. The fluid passes between the gears' surfaces and fixture walls. It is often vertical, but also has motion in the opposite direction (Figure 2(a)). The fluid channel is used to refer to the fluid flowing area. Experimental setup is displayed in Figure 2(b). Hydraulic cylinders situated between the two halves of the MFRP are in turn flanked by two chambers, one filled with a viscous oil and the other filled with viscous MRP media (Das et al., 2010). Hydraulic cylinders provide pressure to MRP media, which in turn is transferred to the workpiece fixture (Figure 3(a)) to push the media to flow over the gear surfaces. Magnets kept in the magnet fixture rotates around the workpiece fixture for uniform finishing of gear (Figure 3 (b)). The schematic diagram of the 
mechanism of MRP fluid flow inside the small gear fixture during the R-MRAFF processing is displayed in Figure 3.. Table 2 describes the gear finishing process factors. Gear has a pitch circle diameter of $12.5 \mathrm{~mm}$, and total teeth is 10 . The MRPF contains IPs, paraffin oil, grease, and abrasive granules, and has the appearance of coarse watery mud (Gopinath et al., 2020). The details of each R-MRAFF process component may be found in Table 1. Abrasive particles of $\mathrm{SiC}$ with a mesh size of 800 are used across the whole of the polishing process.

Table 1 Volume percentage of MRPF constituents

\begin{tabular}{|l|l|l|l|l|}
\hline Constituents & $\begin{array}{l}\text { Iron particles } \\
\text { (IPs) }\end{array}$ & $\begin{array}{l}\text { Abrasive } \\
\text { particles (APs) }\end{array}$ & $\begin{array}{l}\text { Grease } \\
(\mathrm{G})\end{array}$ & $\begin{array}{l}\text { Paraffin } \\
\text { oil (P) }\end{array}$ \\
\hline Volume \% & 22 & 8 & 10 & 60 \\
\hline
\end{tabular}

Table 2 Experimental conditions

\begin{tabular}{|l|l|l|l|l|}
\hline $\begin{array}{l}\text { Experimental } \\
\text { conditions }\end{array}$ & $\begin{array}{l}\text { Finishing } \\
\text { cycles }\end{array}$ & Pressure & $\begin{array}{l}\text { Inlet } \\
\text { velocity }\end{array}$ & $\begin{array}{l}\text { Rotational } \\
\text { speed }\end{array}$ \\
\hline Value & 800 & $32.5 \mathrm{bar}$ & $0.5 \mathrm{~m} / \mathrm{s}$ & $100 \mathrm{rpm}$ \\
\hline
\end{tabular}

Nanometric surface polish is obtained after the 800 polishing cycle. As the experiment is carried out further, it is discovered that several of the surfaces have improved surface finish, but gear teeth surfaces have suffering from increased chipping, and distortion of the gear teeth involute profiles have been detected.

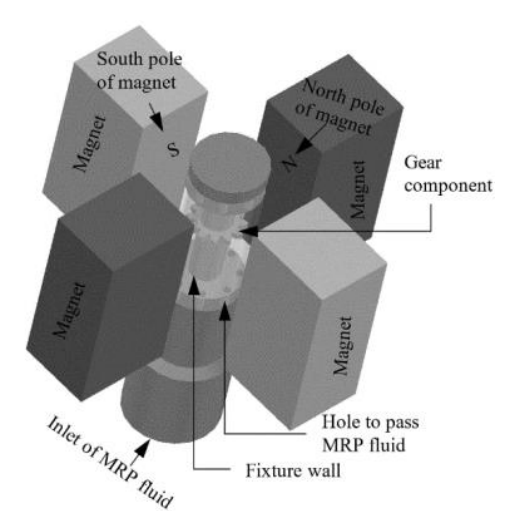

Figure 1 Schematic of 3D computational domain for magnetostatic fluid flow simulation 


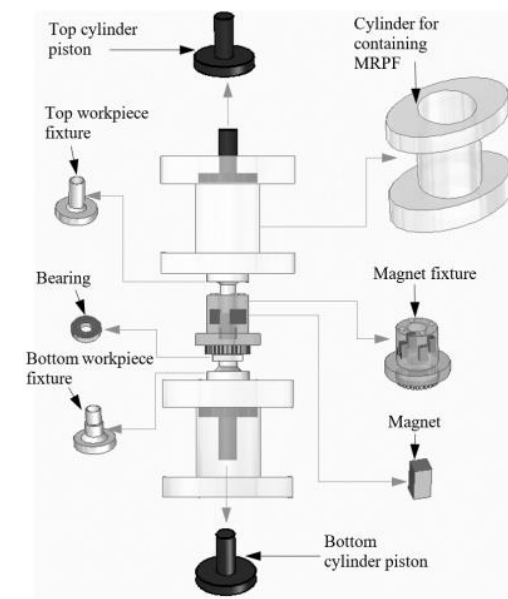

(a)

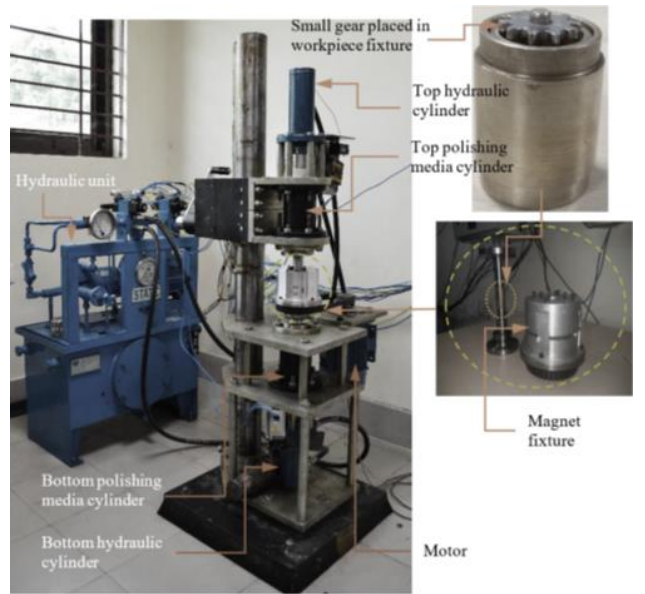

(b)

Figure 2 (a) Schematic view of and (b) pictorial view of R-MRAFF experimental setup with magnet fixture, gear component and workpiece fixture

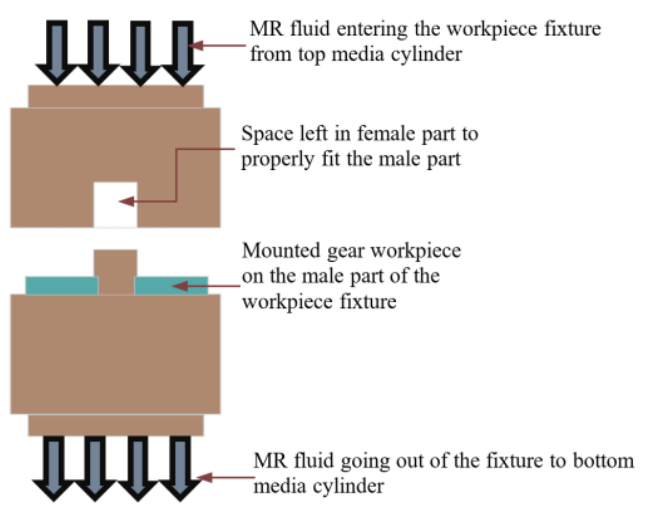

(a)

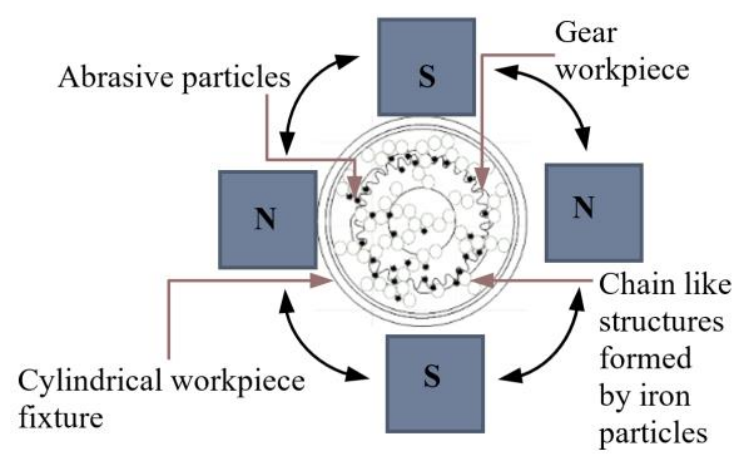

(b)

Figure 3 Flow of MRPF during the polishing process (a) side view and (b) top view

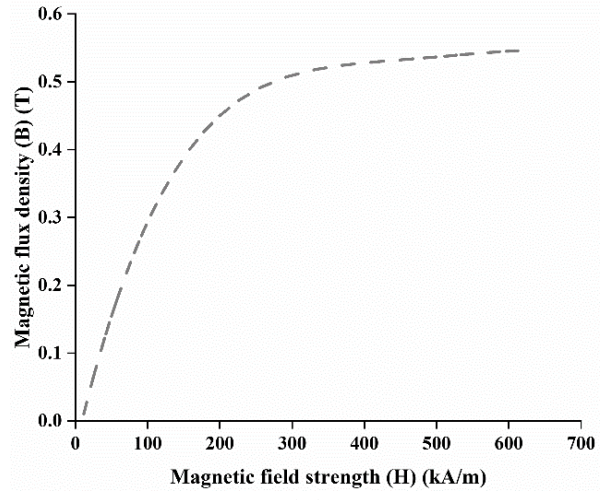

(a)

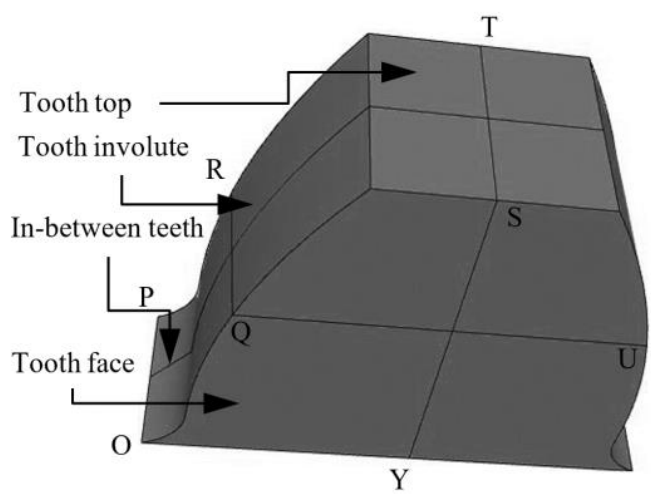

(b)

Figure 4 (a) B-H curve of MRP fluid and (b) chosen locations for variations of magnetic

field and shear stress distribution along gear teeth in-between, top, face \& involute surface 
The fundamental steps in performing any computation in COMSOL® Multiphysics program are as follows: initially, create or purport geometrical model, afterwards insert material, then introduce physics with one's boundaries condition, mesh numerical domain, further introduce study and calculate numerical simulations, and lastly obtain generated outcome. The moving mesh in COMSOL® Multiphysics program is considered for the rotational domain of magnets, and it is given as 100 rpm (Maurya et al., 2020).

\section{Rheological property of MRPF}

Fluid streaming is more strongly influenced by outside factors than fluid channel construction. Understanding the fluid's magnetic characteristics is necessary to regulate the fluid's conduct in the magnetic field. VSM analysis was done in order to get the MB curve, which, in turn, generates the $\mathrm{BH}$ curve. In order to simulate MRP media, the $\mathrm{BH}$ curve is employed in numerical domain. Magnetic field strength may be assessed using this formula:

$$
\begin{gathered}
B=\mu_{0}(H+M) \\
M=\rho M_{m}
\end{gathered}
$$

Experimentation value in Eq. (1) are entered in, resulting in the BH curve in Figure 4(a). Figure 4 (b) displays the locations of setup in relation to the change of shear stress and the magnetic field.

Shear rate fluctuation and changes in the magnetic field induce variations in the flow channel's MRP medium viscosity. This equation is used to determine the MRP media density, a blended mix of all the components:

$$
\rho_{\text {MRP media }}=V_{I P} \rho_{I P}+V_{A P} \rho_{A P}+V_{G} \rho_{G}+V_{P} \rho_{P}
$$

Bingham plastic model, a perfect viscosity model for MRP medium, is used in the Bingham experiment (Gopinath et al., 2020).

In contrast to the Bingham plastic model, the model is really not continuous. In turn, it can't be put to use in calculation because of the difficulty in application. There are ways to fix when you have the occasional gap. The following equations provide a solution for an acceptable discontinuous model that has a high probability of success (Mitsoulis, 2007). 


$$
\tau=\eta \dot{\gamma}
$$

where $\eta$ is the apparent viscosity. Bingham-Papanastasiou model may provide the effective solution of the apparent viscosity.

$$
\eta=\mu+\frac{\tau_{y}}{|\dot{\gamma}|}\left[1-e^{(-m|\dot{\gamma}|)}\right]
$$

where $\mu=$ plastic viscosity (Pa.s), $m$ is consistency index and $\tau_{y}=$ yield stress.

There is a relationship between the magnetic field and yield stress at numerous shear rate, such as $\tau_{y} \propto B^{3}$ and $\mu \propto B^{3}$ (Ginder et al., $1996 \&$ Jayant and Jain, 2019). To conduct research on the rheology of MRPFs, it is necessary to analyze the viscosity of the fluid, which is why a magnetic field related shear stress equation is so significant. When the magnetic field strength is elevated, yield stress will rise. A magnetic field's influence on the yield stress is the inverse of the ratio of diamagnetic to paramagnetic substances (Ginder et al., 1996).

$$
\tau_{y}(H)=\sqrt{6} \phi \mu_{0} M_{s}^{0.5} H_{0}^{1.5}
$$

Where, " $\tau$ " is shear stress, $\eta$ is the viscosity at a high shear rate " $\phi$ " is volume fraction of IPs, " $H_{0}$ " is used magnetic field strength $(\mathrm{A} / \mathrm{m})$, " $M_{s}$ " is saturation magnetization, $\mu_{0}$ is magnetic permeability of free space $\left(4 \pi \times 10^{-7} \mathrm{~N} / \mathrm{A}^{2}\right)$. Here, in simulation, shear stress can be modelled based on Eq.(4).

\section{Governing equations of magnetic field}

The numerical model is being simulated using the magnetic field physics module. The study doesn't need time to run. A BH curve has properties of MRP medium programmed into it, which is then used to conduct the simulation. When it comes to governing magnetic field equation, Maxwell's equation is most often utilised. These are the details of the individuals equations.

$$
B=\nabla \times A
$$

Where, $B$ is magnetic field, and $A$ is magnetic vector potential. Because magnetic monopoles are unable to occur, the divergence of the magnetic field must be zero. 


$$
\nabla \cdot B=0
$$

\section{MRP FLUID FLOW MODELLING}

The fluid flow module has magnetic field module characteristics which are linked to it to mimic the flow of MRPF. Computational domain analysis of a static immiscible, simple laminar fluid is conducted using basic simulation data.

While polishing, there are the forces of gravity and magnetism, but there are also other forces acting on the MRPF. When compared to the gravitational force, the magnetic force is very strong, thus gravity's effect is ignored. Kelvin force, often known as magnetic force. One may use the following to assess this magnetic force unit volume:

$$
F=\mu_{0} v_{i p}(M . \nabla) H
$$

Where, $F$ is force/unit volume $\left(\mathrm{N} / \mathrm{m}^{3}\right), v_{i p}$ is the volume fraction of IPs, and $M$ is magnetization. Following simplification, the equation above may be expressed as follows:

$$
\begin{aligned}
& F_{x}=\mu_{0} v_{i p}\left(M_{x} \cdot \frac{\partial H_{x}}{\partial x}+M_{y} \cdot \frac{\partial H_{x}}{\partial y}+M_{z} \cdot \frac{\partial H_{x}}{\partial z}\right) \\
& F_{y}=\mu_{0} v_{i p}\left(M_{x} \cdot \frac{\partial H_{y}}{\partial x}+M_{y} \cdot \frac{\partial H_{y}}{\partial y}+M_{z} \cdot \frac{\partial H_{y}}{\partial z}\right) \\
& F_{z}=\mu_{0} v_{i p}\left(M_{x} \cdot \frac{\partial H_{z}}{\partial x}+M_{y} \cdot \frac{\partial H_{z}}{\partial y}+M_{z} \cdot \frac{\partial H_{z}}{\partial z}\right)
\end{aligned}
$$

Magnetic field affects fluid flow, and therefore the Navier-Stokes equations includes volume force to model this impact. Eq. (14) includes a term (F) for body force which is defined according to Eq. (13). The following equation provides the magnetic body force that occurs in the MRPF as a consequence.

$$
F=F_{x} \hat{x}+F_{y} \hat{y}+F_{z} \hat{z}
$$

The Navier-Stokes equation takes into consideration the magnetic field's effect on the fluid flow with a volume force. 


$$
\rho(\vec{u} . \nabla) \vec{u}=\nabla \cdot\left[-p I+\eta\left(\nabla \vec{u}+(\nabla \vec{u})^{T}\right)\right]+F
$$

where, $\eta$ is dynamic viscosity $(\mathrm{kg} /(\mathrm{m} . \mathrm{s})), u$ is the velocity $(\mathrm{m} / \mathrm{s}), p$ is the pressure $\left(\mathrm{N} / \mathrm{m}^{2}\right)$. Mesh used for fluid flow simulation was reused to create the magnetic field mesh, which has additional corner and boundary refinement to better-converged results. Another assumption in the model is that fluid flow is independent of time.

\section{RESULTS AND DISCUSSION}

We saw shear stress and magnetic field distributions on gear tooth profile as outputs variables in the simulation. This study implies that the MRP medium's characteristics do not change with time or temperature. Based on the simulation results, a theoretical concept is built in identifying the finishing performance of the MRP media to gear profiles in the R-MRAFF process. Later, experiments are performed, and gear profiles are characterized using the optical profilometer (make: Taylor Hobson) and FESEM analysis.

\section{Magnetic field and shear stress distribution along small gear teeth profiles}

The FE analysis outcomes for variations in magnetic flux distribution along arc length of inbetween (OP), involute (QR), top (ST) and face (QU \& SY) (Figure 4(b)) surface of the gear tooth are shown in Figs. 5(a) (volume plot) and (b), respectively. The maximum magnetic flux density is obtained as $0.25 \mathrm{~T}$ for the top profile of the gear tooth, which is more near to the magnet axis. The results obtained from magnetostatic finite element analysis and literature survey for MR finishing of steel components suggests that this arrangement of magnets and values of magnetic flux density along gear profile is sufficient enough for finishing tiny gear surfaces. This helps to achieve the goal of the present work, such as enhancing surface finishing and eliminating any surface defects. The primary design criteria for all manufacturing processes is the effect of stresses on the workpiece, and the research of characteristics such as shear yield stress of MRP media has been essential since they are a significant design element in applications of MRPFs. Because the quantity of material removed in a finishing operation 
relies heavily on the surface shear stress. Without an understanding of the stress applied to the component surface, it is impossible to provide the high-quality surface finish needed.

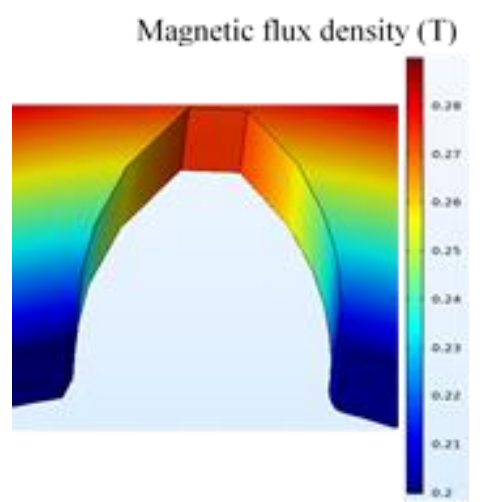

(a)

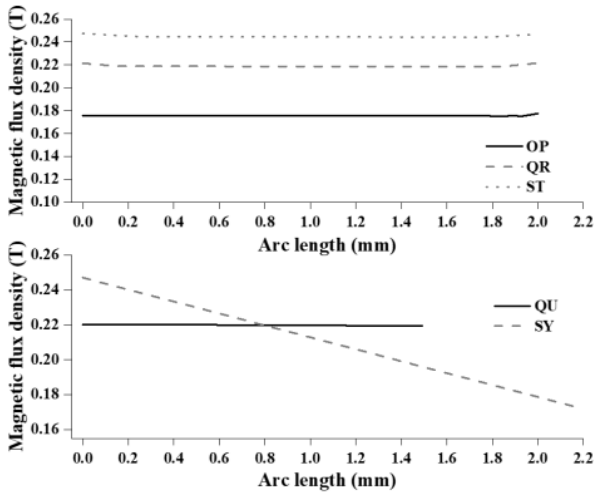

(b)

Figure 5 (a) Volume plot of variations of magnetic flux density along gear teeth profile; (b) magnetic flux density distribution along gear teeth profile

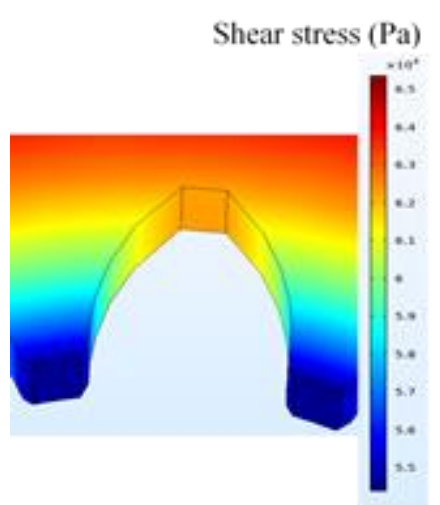

(a)
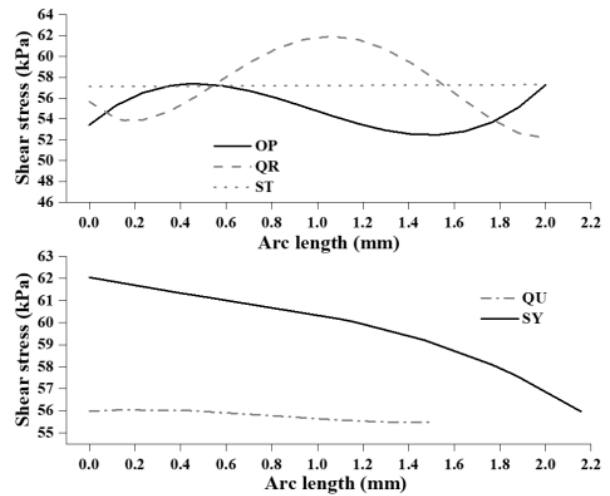

(b)

Figure 6 (a) Volume plot of variation of shear stress along gear teeth profiles; (b) shear stress distribution along the gear teeth profiles

The FEA obtained results for the variation in the distribution of shear stress along the arc length of in-between (OP), an involute (QR), top (ST) and face (QU \& SY) (Figure 4 (b)) surface of the gear tooth are displayed in Figure 6(a) (volume plot) and (b), consecutively. Shear stress at the gear teeth top face is constant, since the magnetic field is homogeneous here due to restricted gap between top profile and cylindrical fixture, meaning that the viscosity of the MRPF remains consistent and is wider, which results in greater values of shear stress. Maximum value of shear stress will result in fast material removal along the gear teeth profile. Maximum shear stress is obtained along gear profiles is $62 \mathrm{kPa}$ (Figure 6 (b)). The characterization of the steel gear workpiece is carried out before and after the finishing 
experiments. Surface roughness and surface topography are assessed using an optical profilometer. FESEM analysis of the workpiece is also carried to observe any recast layer or loose material is still present on involute gear surfaces.

\section{Surface topography and surface roughness analysis of small steel gear component}

Surface topography and surface roughness of gear tooth involute profile pre and post polishing operation are shown in Figure 7. The involute profile before finishing is covered with burrs and scratch marks, as shown in Figure 7(a). After finishing with the R-MRAFF process, burrs and scratch marks are almost removed from the gear profile, as shown in Figure 7(b). The final average surface roughness value $(R a)$ is achieved as $34.5 \mathrm{~nm}$ from the initial $R a$ of $239 \mathrm{~nm}$, as demonstrated in Figs. 7(c) and (d), consecutively. Percentage improvement in surface roughness in the case of the involute profile is $85.56 \%$. Table 3 represents the surface roughness value obtained at various profile surfaces of miniature gear teeth profiles.

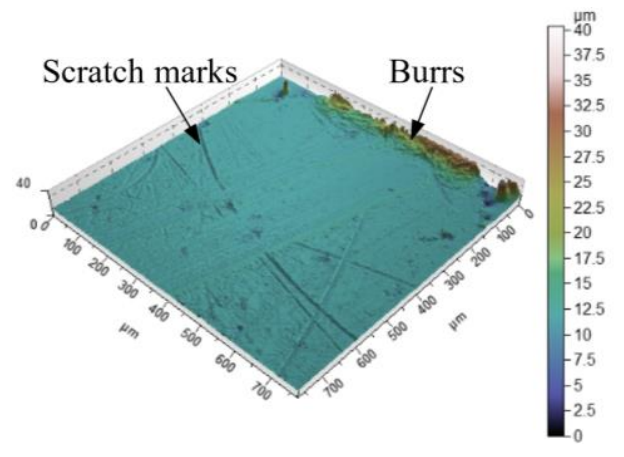

(a)

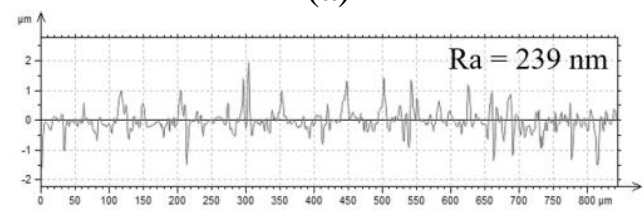

(c)

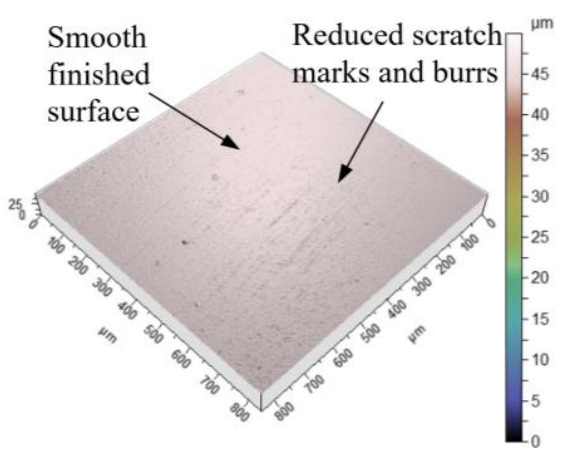

(b)

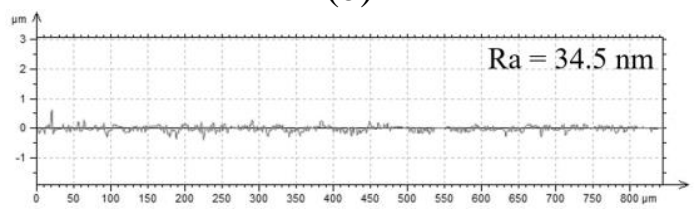

(d)

Figure 7 3D surface topography of gear involute profile (a) pre and (b) post polishing; surface roughness profile of gear involute profile (c) pre and (d) post polishing According to the surface roughness study, percentage improvement in surface roughness is greater in the in-between profile of gear tooth, tooth top profile and involute profile surface than gear tooth face surface. In R-MRAFF method, the face surface of the gear workpiece is subjected to more tangential force than the shear force due to rotational and reciprocating 
movements of MRPF and position of gear workpiece in workpiece fixture during polishing. On the other hand, the involute, tooth top and in-between profile surface of the gear tooth is subjected to more shear force than tangential force. Due to this difference in finishing forces, material removal is higher in the involute surface, tooth top and in-between gear tooth profile than the face surface of the gear tooth. This is acceptable as the gear tooth face is not a working surface. Gear teeth surfaces near the top had the greatest improvement in Ra because greater shear stress occurs there.

Table 3 Measured surface roughness at various profile of gear teeth

\begin{tabular}{|c|c|c|c|}
\hline $\begin{array}{c}\text { Gear tooth } \\
\text { profiles }\end{array}$ & $\begin{array}{c}\text { Initial } \\
\text { Ra }(\mathbf{n m})\end{array}$ & $\begin{array}{c}\text { Final Ra } \\
(\mathbf{n m})\end{array}$ & \% Change in Ra \\
\hline In-between & 396 & 52 & 86.86 \\
\hline Involute & 239 & 34.5 & 85.56 \\
\hline Top & 302 & 34 & 88.74 \\
\hline Face & 206 & 42 & 79.61 \\
\hline
\end{tabular}

\section{FESEM analysis and MRR of steel gear component}

FESEM analysis is used to observe the micrograph images of the gear workpiece before and after finishing. Micrograph analysis helps to observe and analyze different kind of defects in working areas of gear workpiece. The surface finish on the involute surface of the gear tooth is observed, and it is found that the surface finish on the involute tooth profile is better after finishing, as shown in Figure 8. The steel gear workpiece is manufactured using a wire EDM process for which it is subjected to recast layers. The presence of the recast layer is confirmed in the involute profiles of the gear tooth (Figure 8(a)) before the finishing process. Following the polishing, recast layer and the loosened material on involute profile are entirely eliminated, as displayed in Figure 8(b). Also, as displayed in Figure 8(b), final surface is smoother than the initial surface. 


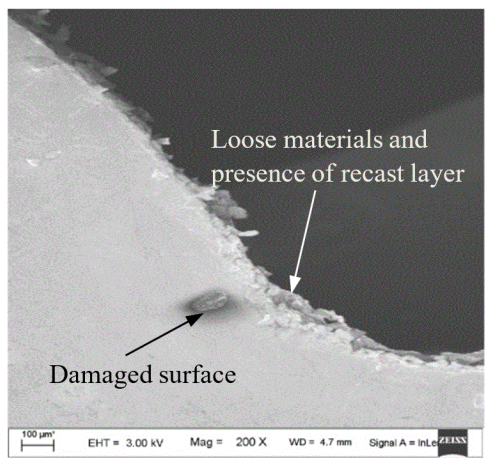

(a)

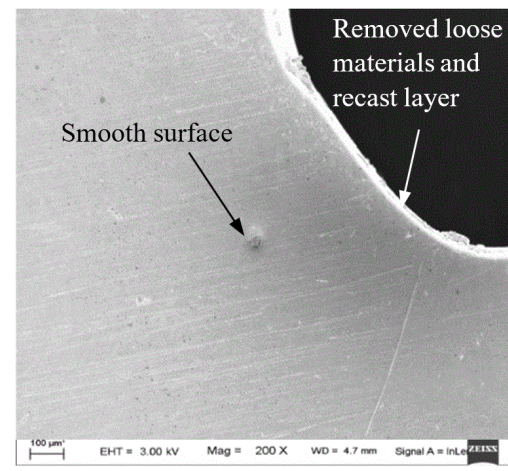

(b)

Figure 8 FESEM image of gear involute profile (a) pre and (b) post polishing

The material removal rate is the total material that is removed per minute. In the present case, the required time to complete the finishing experiment is 210 minute. The weight of the gear component before and after finishing is measured as $1.6783 \mathrm{gm}$ and $1.5870 \mathrm{gm}$, respectively. The final MRR ((initial weight - final weight) / finishing time) obtained is $0.0004347 \mathrm{gm} / \mathrm{min}$.

\section{CONCLUSIONS}

The COMSOL ${ }^{\circledR}$ Multiphysics program is used to conduct the magnetostatic fluid flow study for the gear R-MRAFF processing. In order to do this CFD study in the applied magnetic field, a 3D numerical model is created. The different flow factors are monitored in order to determine the conduct of the MRPF throughout the polishing. This simulation is being carried out in order to get a better understanding of the R-MRAFF processing. Viscosity model for MRPF based on the Bingham-Papanastasiou equation is used in this study. The magnetic field has an effect on viscosity and yield stress of MRPF. A greater finishing rate will be achieved when the fluid flow path cross-section is at its smallest. Surface roughness has been improved the most on the top surface profile of the gear component, which is where shear stress is at its greatest, resulting in quicker material removal and finishing rates. The final surface roughness obtained at the involute gear profile is " $\mathrm{Ra}=34.5 \mathrm{~nm}$ ", and also all manufacturing defects have been removed. The experimentally calculated material removal rate (MRR) obtained for gear profiles are $0.0004347 \mathrm{gm} / \mathrm{min}$. All manufacturing defects have been removed from the small gear surface 
profiles. It is possible that a fine uniform surface finishing on gear profiles would enhance the practical uses of the tiny gear and also resulting in a prolonged lifespan with no meshing errors.

\section{ACKNOWLEDGMENT}

We acknowledge the Science \& Engineering Research Board (SERB), New Delhi, India, for their financial support for project No. EEQ/2017/000597 entitled "Fabrication of Prosthetic Im-plants and further Nanofinishing using Magnetic Field Assisted Finishing (MFAF) Process".

\section{REFERENCES}

Alaskari, Liptakova, T., Fajnor, P., \& Halamova, M. 2014. Mechanical surface treatments effects on corrosion of AISI 316 Ti stainless steel in chloride environments. Journal of Engineering Research 2(3): 195-210.

Das, M., Jain, V. K., \& Ghoshdastidar, P. S. 2010. Nano-finishing of stainless-steel tubes using rotational magnetorheological abrasive flow finishing process. Machining Science and Technology 14(3): 365-389.

Ginder, J. M., Davis, L. C., \& Elie, L. D. 1996. Rheology of magnetorheology fluids: models and measurements. International Journal of Modern Physics 10(23): 11.

Gopinath, B., Sathishkumar, G. K., Karthik, P., Martin Charles, M., Ashok, K. G., Ibrahim, M., \& Mohamed Akheel, M. 2020. A systematic study of the impact of additives on structural and mechanical properties of Magnetorheological fluids. Materials Today: Proceedings.

Jayant, \& Jain, V. K. 2019. Analysis of finishing forces and surface finish during magnetorheological abrasive flow finishing of asymmetric workpieces. Journal of Micromanufacturing 2(2): 133-151.

Kumar, M., Kumar, A., Alok, A., \& Das, M. 2020. Magnetorheological method applied to optics polishing: A review. IOP Conference Series: Materials Science and Engineering 804(01): 12-13.

Kumar, M., Yadav, H. N. S., Kumar, A., \& Das, M. 2021. An overview of magnetorheological polishing fluid applied in nano-finishing of components. Journal of Micromanufacturing 3(5): 1-19.

Maurya, N. K., Rastogi, V., \& Singh, P. 2020. Experimental and computational analysis of 
mechanical properties of RGD840 material manufactured through PolyJet process. Rapid Prototyping Journal 27(1): 207-214.

Mitsoulis, E. 2007. Flows of viscoplastic materials: models and computations. In Rheology Reviews.

Nagdeve, L., Jain, V. K., \& Ramkumar, J. 2018. Nanofinishing of freeform/sculptured surfaces: state-of-the-art. Journal of Manufacturing Review 6(3): 1-20.

Soler, Y. I., \& Le Nguyen, V. 2018. Selection of abrasive wheels by surface topography of parts from hardened steel 30ChGSA. Journal of Engineering Research 6(2): 191-208.

Yi, J., Ding, Y., Zhao, S., Ji, B., \& Zhou, J. 2009. A novel technique of polishing gear working surface using PECMP. International Journal of Precision Engineering and Manufacturing 10(4): 57-62. 